\title{
Ultraviolet Durable Flexible Nonfullerene Organic Solar Cells Realized by a Hybrid Nanostructured Transparent Electrode
}

Tao Xu ${ }^{1}$, Chunliu Gong ${ }^{1}$, Shuanglong Wang ${ }^{1}$, Hong Lian ${ }^{1}$, Weixia Lan ${ }^{1}$, Gaëtan Lévêque ${ }^{2}$, Bruno Grandidier ${ }^{2}$, Jerôme Plain $^{3}$, Renaud Bachelot ${ }^{3}$, Bin Wei ${ }^{{ }^{*}}$, Furong Zhu ${ }^{4 *}$

${ }^{1 .}$ School of Mechatronic Engineering and Automation, Key Laboratory of Advanced Display and System Applications, Ministry of Education, Shanghai University, 200072, Shanghai, China

${ }^{2 .}$ IEMN, UMR8520, Université de Lille 1, 59652 Villeneuve d'Ascq Cédex, France

${ }^{3 .}$ Light, nanomaterials, nanotechnologies (L2n) Laboratory. Charles Delaunay Institute, CNRS. University of Technology of Troyes, 12 rue Marie Curie, F-10004 Troyes Cedex, France

${ }^{4}$ Department of Physics, Research Centre of Excellence for Organic Electronics and Institute of Advanced Materials, Hong Kong Baptist University, Kowloon Tong, Hong Kong, China

*Corresponding authors

Email: bwei@shu.edu.cn, frzhu@hkbu.edu.hk 


\begin{abstract}
A significant enhancement in ultraviolet (UV) durable indium tin oxide (ITO)-free flexible nonfullerene organic solar cells (OSCs) has been demonstrated using a hybrid nanostructured flexible transparent electrode (FTE), comprising a mixture of OD silver nanoparticles (AgNPs), 1D Ag nanowires (AgNWs) and 2D exfoliated graphene sheets. The FTE exhibits high optical transparency and electric conductivity, good air stability and full-solution fabrication capability at a low processing temperature. An average power convention efficiency (PCE) of $8.15 \%$ has been obtained for the flexible nonfullerene OSCs, based on the blend of poly[(2,6-(4, 8-bis(5-(2-ethylhexyl)thiophen-2-yl)-benzo[1,2-b:4,5-b']dithiophene))-alt-(5,5-(1',3'-di-2-thi enyl-5',7'-bis(2-ethylhexyl)benzo[1',2'-c:4',5'-c'] dithiophene-4,8-dione)] (PBDB-T): 3,9bis(2-methylene-(3-(1,1-dicyanomethylene)-indanone))-5,5,11,11-tetrakis(4-hexylphenyl)-dit hieno[2,3-d:2',3'-d']-s-indaceno[1,2-b:5,6-b'] dithiophene (ITIC). The flexible PBDB-T:ITIC OSCs exhibit an excellent UV durability compared to the ITO-based control cell, realized by the tailored FTE absorption in wavelength $<380 \mathrm{~nm}$. The novel FTE developed in this work provides a promising alternative to ITO for use in UV durable flexible OSCs, serving as a UV filter to impede an unavoidable UV-induced degradation in ITO-based OSCs.
\end{abstract}

Keywords: UV durability, flexible organic solar cells, flexible transparent electrode, silver nanowires, hybrid nanostructures 


\section{Introduction}

The development of high performance flexible nonfullerene organic solar cells has attracted considerable attention because of their advantages of high efficiency, mechanical flexibility, and large area solution-fabrication capability at a low cost. ${ }^{1-5}$ Flexible transparent electrodes (FTEs) with superior electric conductivity, high optical transparency and mechanical flexibility are the prerequisites for application in flexible OSCs. ${ }^{6-8}$ Indium-tin-oxide (ITO) is a widely used transparent electrode, but it is rigid and cannot be used in flexible OSCs. ${ }^{9}$ Various types of FTEs have been proposed to replace ITO such as: metallic nanostructures (e.g., nanowires, grids), ${ }^{10,11}$ carbon-based materials (e.g., nanotubes, graphene) ${ }^{12,13}$ and conducting polymers. ${ }^{14}$ Among them, a layer of silver nanowires (AgNWs) is one of the most promising candidates as an alternative FTE for use in flexible optoelectronic devices because of its superior optical and electric properties. ${ }^{15-17}$ However, AgNW-based FTEs still face some technical challenges: first, the randomly distributed AgNWs formed during the solution process result in a large surface roughness that may penetrate through the active layer, leading to electric shorts; second, percolation of charges through junctions between AgNWs results in large contact resistance. Moreover, large tensile deformations lead to the detachment of the NWs at the junctions, which cannot be recovered upon relaxation of the deformation. As a result, the production of highly conductive and flexible AgNW-based electrode is still one of the open challenges for efficient OSCs. Different approaches have been applied to address this issue. For instance, AgNWs were embedded in the polymer matrix that were further processed with thermal annealing or high-force pressing to reduce the surface roughness. ${ }^{18,19}$ Different types of nanoparticles (NPs) were added in the NW network to fill the empty spaces between the AgNWs, leading to a decrease in the sheet resistances $(R s){ }^{20,21}$ FTEs incorporating two-dimensional (2D) graphene sheets in AgNW-based FTEs were proposed. The use of graphene sheets helps to reduce the surface roughness and improves the mechanical stability, taking advantage of the $2 \mathrm{D}$ graphene pathways for enhancing charge transfer and stretchability of graphene. ${ }^{22-25}$ The application of such FTEs has made some initial progresses in the flexible OSCs, however, more improvement is needed. 
In parallel to enhance the operation stability of the OSCs, the UV durability is another critical factor for applications in flexible OSCs. ${ }^{26-28}$ Deterioration in the performance of the flexible OSCs due to the moisture encroachment and oxidation at the organic electrode interfaces can be minimized by proper encapsulation. The use of the graphene sheets, serving as an oxidation-resistant layer, helps to protect $\mathrm{AgNWs}$, and thereby improving the long-term stability of AgNWs-based FTEs. ${ }^{29,30}$ It is well known that OSCs are unstable under UV irradiation, ${ }^{31,32}$ however, the development of UV durable flexible nonfullerene OSCs has not yet been systemically studied.

In this work, we report our effort to develop a high performance UV durable flexible nonfullerene OSCs realized by incorporating a novel hybrid nanostructured FTE, comprising a mixture of 0D AgNPs, 1D AgNWs and 2D exfoliated graphene sheets. The all-solution processable hybrid nanostructured FTE has a low sheet resistance of $24 \mathrm{ohm} \mathrm{sq}^{-1}$, a high optical transparency of $82 \%$ over the visible light wavelength range and a superior mechanical flexibility. The flexible OSCs, made with a blend system of poly[(2,6-(4, 8-bis(5-(2-ethylhexyl)thiophen-2-yl)-benzo[1,2-b:4,5-b']dithiophene))-alt-(5,5-(1',3'-di-2-thi enyl-5',7'-bis(2-ethylhexyl)benzo[1',2'-c:4',5'-c'] dithiophene-4,8-dione)] (PBDB-T): 3,9bis(2-methylene-(3-(1,1-dicyanomethylene)-indanone))-5,5,11,11-tetrakis(4-hexylphenyl)-dit hieno[2,3-d:2',3'-d']-s-indaceno[1,2-b:5,6-b'] dithiophene (ITIC), possess a high power conversion efficiency (PCE) of $8.15 \%$ and retain $>90 \%$ of the initial PCE after a test of 1000 bends at a radius of $4.0 \mathrm{~mm}$. A significant enhancement in the UV stability of the flexible OSCs has been obtained under a UV-assisted acceleration aging test, arising from the tailored absorption of the hybrid nanostructured FTEs in wavelength $<380 \mathrm{~nm}$.

\section{Results and Discussions}

A schematic fabrication process of the hybrid AgNWs:AgNPs/graphene FTE is shown in Figure 1(a). A layer of AgNWs:AgNPs was first coated on the poly(methyl methacrylate) (PMMA)-coated polyethylene terephthalate (PET) substrate, forming a network of AgNWs:AgNPs embedded in the PMMA, which was flattened by a post mechanical pressing process. A layer of exfoliate graphene sheets was then overlaid on the surface of the AgNWs:AgNPs matrix forming a hybrid AgNWs:AgNPs/graphene FTE. 
The scanning electron microscopy (SEM) and atomic force microscopy (AFM) images measured for the surface of AgNWs:AgNPs embedded in the PMMA are shown in Figures 1(b) and 1(c). It shows that the AgNWs with a diameter of $80 \mathrm{~nm}$ and a length of 20-30 $\mu \mathrm{m}$ were randomly distributed forming an AgNW network. AgNPs with a diameter of $60 \mathrm{~nm}$ distributed randomly across the AgNWs, as shown in Figure 1(b). In a related work, we have demonstrated that incorporation of gold NPs in the hole transporting layer (HTL) helps to improve the adhesion at the anode/organic interface, and also helps to enhance the charge extraction and reduce the leakage current by improvement of contact property. ${ }^{33}$ The use of the AgNPs is to enhance the adhesion between the exfoliate graphene (EG) sheets and improves the electric conductivity of the hybrid nanostructured FTEs. Another important parameter of the FTEs for use in flexible OSCs is the surface roughness. In this work, the AgNWs:AgNPs were partially embedded in PMMA with a root-mean-square (RMS) roughness of $\sim 17.2 \mathrm{~nm}$, which is obviously lower than that of the pristine AgNWs formed on PET substrate (RMS $28.5 \mathrm{~nm}$ ). Some pinholes, seen in Figure 1c and Figure S1 in Supporting Information (SI), were observed on the surface of AgNWs:AgNPs embedded PMMA, which were formed due to the solvent evaporation during the annealing process.

A layer of EG sheets was coated on the AgNWs:AgNPs surface to enhance the mechanical flexibility of FTEs. Comparing to the graphene grown by CVD, ${ }^{34}$ EG dispersion has advantages of low-cost, solution-processing and large-scale production, a promising process option for application in flexible OSCs. EG layer also has a lower density of defects as compared to that of the reduced graphene oxide (rGO), leading to an improved electric conductivity. ${ }^{35}$ In this work, the quality of the graphene layer was evaluated and optimized by forming an EG layer using spray coating. Spray coating was adopted because of its potential for producing large area uniformity graphene film. ${ }^{36}$ SEM and AFM images measured for the EG films deposited on ITO substrates are shown in Figure S1 in SI, showing a smooth surface with a RMS of $<3.7 \mathrm{~nm}$. Raman spectra measured for the EG film are shown in Figure 1(d), the intensity of the $\mathrm{G}$ peak $\left(\sim 1580 \mathrm{~cm}^{-1}\right)\left(\mathrm{I}_{\mathrm{G}}\right)$ is much higher than that of the D peak $\left(\mathrm{I}_{\mathrm{D}}\right)$. The ratio of $I_{D}$ to $I_{G}\left(I_{D} / I_{G}\right)$ is 0.24 , which is much smaller than that measured for an rGO layer (1.1-1.5). ${ }^{37}$ Moreover, the $2 \mathrm{D}$ peak at a wavenumber of $\sim 2700 \mathrm{~cm}^{-1}$ is clearly visible. This 
suggests that the EG layer thus prepared has a high graphene quality, despite the presence of a small defect-induced D peak $\left(\sim 1350 \mathrm{~cm}^{-1}\right)$, caused by edges of the graphene flakes. ${ }^{38}$ A small ratio of $\mathrm{I}_{\mathrm{D}} / \mathrm{I}_{\mathrm{G}}$ of 0.22 was obtained for AgNWs:AgNPs/graphene FTEs, confirming the high quality EG film on AgNWs:AgNPs. Compare to the pristine EG film, the enhanced peak intensity in the Raman spectra, measured for AgNWs:AgNPs/graphene, is closely associated with the surface enhanced Raman scattering induced by Ag nanostructures. ${ }^{39}$ AFM image measured for the AgNWs:AgNPs/graphene is shown in Figure 1(e). It reveals that EG sheets are uniformly overlaid on the AgNW network, resulting in a low RMS of $6.6 \mathrm{~nm}$ as compared to that of the AgNWs:AgNPs surface (RMS $17.2 \mathrm{~nm}$ ). The height of AgNWs also decreases significantly, as illustrated by the white circles in Figures 1(d) and 1(e). SEM images measured for the AgNW samples with full and partial EG coverages are shown in Figure S2 in SI. AFM and SEM measurements reveal clearly that the EG sheets were uniformly coated on the surface of the AgNWs:AgNPs interpenetrating networks. The hybrid AgNWs:AgNPs/graphene FTE with a smooth surface is favorable for achieving high performance flexible OSCs.

There is an inverse correlation between $R s$ and the optical transparency of AgNW-based FTEs, which is highly dependent on the fabrication process. ${ }^{40}$ The optical transparency and $R s$ measured for different hybrid nanostructured FTEs are shown in Figures 2(a) and 2(b). An optimal FTE of the bare AgNWs embedded in PMMA had a $R_{s}$ of $47 \mathrm{ohm} \mathrm{sq}{ }^{-1}$ and an average transparency of $\sim 87 \%$ over the visible light wavelength range from 400 to $800 \mathrm{~nm}$. A slightly lower average transparency of $86 \%$ and a lower $R_{s}$ of $33 \mathrm{ohm} \mathrm{sq}{ }^{-1}$ were observed when the AgNPs were incorporated in the AgNWs-based FTEs. The visible light transparency of the hybrid AgNWs:AgNPs FTE decreased to $\sim 81 \%$ after a uniform EG layer was coated on its surface, leading to a further decrease in $R_{S}\left(23 \mathrm{ohm} \mathrm{sq}^{-1}\right)$. It is anticipated that the use of a few (2-3) layers of the 2D EG sheets, helps to bridge the empty spaces between the AgNWs, leading to an obvious increase in the lateral conductivity and a slight decrease in the optical transparency of the hybrid nanostructured FTEs. To better assess the performance of the transparent electrode, the figure of merit $(\mathrm{FoM})$, defined as the ratio of the electric conductivity to optical transparency, is used for analyzing the optical and electric properties of 
the FTEs. The value of the FoM of the FTEs is calculated using the following equation: ${ }^{41}$

$$
\mathrm{FoM}=\frac{188.5}{R s\left(T(\lambda)^{-0.5}-1\right)}
$$

where $T(\lambda)$ is the optical transparency of the FTEs measured at $550 \mathrm{~nm}$. The FoM values of 57 , 71 and 79 are obtained for different FTEs of AgNWs, AgNWs:AgNPs and AgNWs:AgNPs/graphene, calculated using equation (1), the results are plotted in Figure 2(b).

It is clear that an optimized hybrid nanostructured AgNWs:AgNPs/graphene FTE possesses the highest FoM, revealing its suitability for application in flexible OSCs. The mechanical flexibility of the hybrid nanostructured FTEs was examined over 1000 bending cycles, with a radius of $4.0 \mathrm{~mm}$, in the bending test. The ratio of $R_{s}$ to the initial sheet resistance $\left(R_{0}\right)$ of $R_{S} / R_{0}$ as a function of the bending cycles and aging time was characterized for the hybrid nanostructured FTEs with and without the EG over layer. The ratios of $R_{s} / R_{0}$ as a function of the number of the bending cycles measured for different FTEs are plotted in Figure 2(c). It shows that $R_{S} / R_{0}$ value measured for the AgNWs was 4.7 times higher than that obtained for the AgNWs:AgNPs/graphene over a test of 1000 bends. A 56\% increase in the $R_{s}$ of an AgNWs/graphene FTE was observed as compared to that of an AgNWs:AgNPs/graphene FTE after a test of 1000 bends. It reveals that the AgNPs serve as a binding medium assisting in enhancing the adhesion between the AgNWs and graphene sheets in the FTEs. The adhesion between graphene and AgNWs with and without the presence of AgNPs was analyzed using different AFM tip-surface contact forces. ${ }^{42}$ In the AFM nanoscale adhesion measurement, AFM tip measures the pull-off force at the graphene/AgNW network interface, as illustrated in Figure S3 in SI, while the adhesion is estimated using the pull-off forces. An adhesion force of $20.2 \mathrm{nN}$ was observed for the AgNWs:AgNPs/graphene FTE, which is $>25 \%$ stronger than the one between graphene and AgNWs in the AgNWs /graphene FTE, as shown in Figure S3 in SI. The AFM adhesion measurements support our previous results in showing that the addition of the metal $\mathrm{NPs}^{33}$ helps to enhance the adhesion between graphene sheets and AgNWs in the hybrid nanostructured FTEs.

The stability of the hybrid nanostructured FTEs was analyzed by monitoring the change 
in the ratios of $R_{S} / R_{0}$ of the FTEs as a function of the aging time, aged over a period of 30 days in air, as shown in Figure 2(d). The advantages of incorporation of EG sheets and AgNPs in the hybrid FTEs are clearly manifested. The AgNWs-based FTE suffers from an unavoidable oxidation of the AgNWs in air resulting in an increase in $R_{s}$. In contrast, the AgNWs:AgNPs/graphene FTEs had a negligible change in $R_{s}$ during the aging test. It is clear that the use of an upper EG layer acts as an effective barrier layer to prevent the encroachment of the moisture and oxygen suppressing the oxidation of AgNWs. SEM images measured for the AgNWs FTEs also support above discussion, revealing the broken AgNW segments in the bare AgNWs-based FTE due to an unavoidable oxidation of the AgNWs, as shown in Figure S4 in SI. In comparison, AgNWs in AgNWs:AgNPs/graphene remained the same morphology. Our results suggest that the incorporation of the EG sheets and AgNPs in the hybrid FTEs has two advantages: (1) it helps to improve the barrier properties, and (2) it enhances the adhesion between the AgNWs and EG sheets, resulting in the hybrid nanostructured AgNWs:AgNPs/graphene FTEs with superior flexibility and electric conductivity.

Absorption spectra measured for the different layers of AgNWs, AgNWs:AgNPs, AgNWs:AgNPs/graphene, PBDB-T:ITIC and ITO are shown in Figure 3. It becomes clear that the hybrid nanostructured AgNWs:AgNPs/graphene FTE has a strong absorption over a short wavelength range with a peak located at $\sim 360 \mathrm{~nm}$, as compared to that of the ITO/glass substrate. The AgNWs:AgNPs/graphene FTE has a weak absorption over the visible light wavelength range from $480 \mathrm{~nm}$ to $720 \mathrm{~nm}$. The absorption profile of the AgNWs:AgNPs/graphene FTE matches well with the absorption of PBDB-T:ITIC blend layer. It allows the visible light passing through for maximum absorption in the organic active layer, and prevents the UV part of the incident light entering to the organic layer. The UV absorption behaviors of the pristine AgNW and AgNW:AgNP were analyzed using Comsol Multiphysics, as shown in Figure S5(a) in SI. The distribution of the electric field amplitude, calculated by Comsol Multiphysics, are plotted in Figure S5(b) in SI. The theoretical simulation agrees with the experimental results, revealing the nature of $\mathrm{AgNW}$ and AgNW:AgNP having a high UV absorption in the wavelength range from $350 \mathrm{~nm}$ to $400 \mathrm{~nm}$. The AgNWs:AgNPs/graphene FTE has an additional advantage serving as a built-in UV filter for improving the UV durability of the flexible OSCs. 
To investigate the feasibility of hybrid nanostructured FTEs for application in flexible OSCs, a set of flexible OSCs comprising a layer configuration of PET/FTEs/poly (3, 4-ethylenedioxy-thiophene) doped with poly (styrenesulfonate) (PEDOT:PSS) (40 nm)/PBDB-T:ITIC (100 nm)/8-hydroxyquinolinolato-lithium (Liq) (1 nm)/Al (100 nm) was fabricated. A $40 \mathrm{~nm}$ thick PEDOT:PSS HTL was used to assist in the hole extraction. The use of the bilayer Liq/Al cathode was to improve the electron collection. The flexible OSCs had a $100 \mathrm{~nm}$ thick PBDB-T:ITIC bulk heterojunction (BHJ) active layer, with an effective active area of $4 \mathrm{~mm}^{2}$. The current density-voltage $(J-V)$ characteristics measured for the flexible OSCs with different FTEs, under AM1.5G $\left(100 \mathrm{~mW} / \mathrm{cm}^{2}\right)$, are plotted in Figure 4(b). A summary of the short circuit current density $\left(J_{s c}\right)$, open circuit voltage $\left(V_{o c}\right)$, fill factor (FF) and PCE is listed in Table 1. The control OSC comprising a layer configuration of glass/ITO/PEDOT:PSS (40 nm)/PBDB-T:ITIC (100 nm)/(Liq) $(1 \mathrm{~nm}) / \mathrm{Al}(100 \mathrm{~nm})$ was also fabricated for comparison study.

The flexible OSCs with a bare AgNWs FTE had an average PCE of 7.2\%, $J_{s c}$ of 12.35 $\mathrm{mA} \mathrm{cm}{ }^{-2}, V_{o c}$ of $0.89 \mathrm{~V}$, and FF of $65.64 \%$. The one with an AgNWs:AgNPs FTE had an increased PCE of $7.66 \%$, with $J_{s c}$ of $12.74 \mathrm{~mA} \mathrm{~cm}{ }^{-2}, V_{o c}$ of $0.88 \mathrm{~V}$ and FF of $67.96 \%$. The improvements in the cell performance are closely associated with the use of the AgNWs:AgNPs FTE, which has a higher conductivity as compared to that of the AgNWs FTE. The average PCE of $8.15 \%$ was obtained for the flexible OSCs with an AgNWs:AgNPs/graphene FTE, along with a $J_{s c}$ of $13.36 \mathrm{~mA} \mathrm{~cm}^{-2}$, a $V_{o c}$ of $0.89 \mathrm{~V}$, and a FF of $68.55 \%$. An obvious enhancement in the cell performance agrees well with the discussion made with the unique features of the hybrid nanostructured AgNWs:AgNPs/graphene FTE, demonstrating its advantages for application in flexible OSCs. A slight deviation in $V_{o c}$ in different OSCs was observed, which is within the experimental error for cells prepared in different batches. The performance of the flexible OSCs made with the solution-processed AgNWs:AgNPs/graphene FTE is comparable to that of the control cell with an ITO anode, as shown in Table 1.

The external quantum efficiency (EQE) spectra measured for the flexible OSCs with 
different FTEs are plotted in Figure 4(c). A broadband enhancement in EQE is observed in the flexible OSCs with an AgNWs:AgNPs/graphene FTE as compared to the ones made with AgNWs and AgNWs:AgNPs FTEs. The EQE results are consistent with the $J-V$ measurements. The maximum $J_{s c}$ of $13.17 \mathrm{~mA} \mathrm{~cm}{ }^{-2}, 12.36 \mathrm{~mA} \mathrm{~cm}{ }^{-2}$ and $11.53 \mathrm{~mA} \mathrm{~cm}$, calculated using the EQE spectra measured for the flexible OSCs with different FTEs of AgNWs:AgNPs/graphene, AgNWs:AgNPs and AgNWs, are presented in Figure 4 (c). The calculated $J_{s c}$ values agree well with the ones obtained by the $J-V$ measurements. These results demonstrate that the solution-processable hybrid nanostructured AgNWs:AgNPs/graphene FTE is a favorable alternative to ITO for use in high performing OSCs.

The photocurrent density $\left(J_{\mathrm{ph}}\right)$ as a function of the effective voltage $\left(V_{\text {eff }}\right)$, as shown in Figure 4(d), was used to analyze the charge extraction in the flexible OSCs prepared using different FTEs, where $J_{\mathrm{ph}}=J_{1}-J_{\mathrm{d}}, J_{1}$ is current density measured under the illumination of AM1.5G, and $J_{\mathrm{d}}$ is the dark current density. The effective voltage $V_{\text {eff }}=V_{0}-V$, where $V_{0}$ is the built-in potential and $V$ is the applied bias. Under a low $V_{\text {eff }}(<0.2 \mathrm{~V})$, the charge recombination increases with decrease in $V_{\text {eff, }}$, therefore not all the photo-generated charge carriers can be collected due to the bimolecular recombination. ${ }^{43}$ Under a high $V_{\text {eff }}(>0.6 \mathrm{~V})$, almost all the photo-generated charge carriers can be swept out and collected, generating a saturated photocurrent density $\left(J_{\mathrm{sat}}\right)$. Apparently, $J_{\mathrm{ph}}$ measured for the flexible OSCs with an AgNWs:AgNPs/graphene FTE can reach saturation under a lower applied bias as compared to the devices with an AgNWs FTE or AgNWs:AgNPs FTE, as shown in Figure S6 in SI, indicating a more efficient charge extraction capability in the flexible OSCs made with an AgNWs:AgNPs/graphene FTE. The charge collection efficiency $P_{C C}$ can be analyzed using the ratio of $J_{\mathrm{ph}} / J_{\text {sat. }}$. Under $V_{\text {eff }}<0.3 \mathrm{~V}$, a higher $P_{\mathrm{CC}}$ is observed for flexible OSCs with an AgNWs:AgNPs/graphene FTE as compared to the cells with a pristine AgNWs contact or a hybrid AgNWs/graphene FTE, as shown in Figure 4(d).

The mechanical flexibility of the flexible OSCs made with different hybrid nanostructured FTEs of AgNWs:AgNPs and AgNWs:AgNPs/graphene was studied by a 
bending test with a radius of $4.0 \mathrm{~mm}$, as it is shown in Figure 5. The $V_{o c}, J_{s c}$ and FF of the flexible OSCs with an AgNWs:AgNPs/graphene FTE can retain > 96\% of their initial values after a test of 1000 bends. While the $V_{o c}, J_{s c}$ and FF of the flexible OSCs with an AgNWs:AgNPs FTE remained only $80 \%-85 \%$ of their initial values, the corresponding PCE of the flexible OSCs with an AgNWs:AgNPs/graphene FTE maintained > 90\% of their initial efficiency, which is much higher than that of the ones made with an AgNWs:AgNPs FTE (< $60 \%$ ). The flexible OSCs with an average PCE of $>8 \%$ are clearly demonstrated.

In a recent work, we have shown that maintaining a stable and high built-in potential across BHJ through interfacial modification favors the efficient and stable operation of OSCs. ${ }^{44,45}$ In addition to the operational stability, the UV durability is another important factor for practical application of OSCs. The UV durability of the flexible OSCs and a control OSC made with an ITO transparent anode was analyzed by exposing the cells to a UV lamp, with a peak wavelength of $360 \mathrm{~nm}$ and an intensity of $200 \mathrm{~W} / \mathrm{m}^{2}$, under different UV exposure times. To avoid the possible cell degradation due the moisture and oxygen encroachment, the accelerated UV durable tests of the flexible OSCs were conducted in the $\mathrm{N}_{2}$-purged glove box with $\mathrm{O}_{2}$ and $\mathrm{H}_{2} \mathrm{O}$ levels $<0.1 \mathrm{ppm}$. The variation in $V_{o c}, J_{s c}, \mathrm{FF}$ and PCE measured for the flexible OSCs under different UV exposure times are compared to that of the control cell, and the results are plotted in Figure 6. Although the ITO-based control cell had a higher initial PCE, a fast decrease in its $V_{o c}, J_{s c}, \mathrm{FF}$ and PCE was observed over a UV exposure period of $60 \mathrm{~min}$. For example, a loss in $V_{o c}$ from $0.89 \mathrm{~V}$ to $0.84 \mathrm{~V}$, a decrease in $J_{s c}$ from $15.45 \mathrm{~mA}$ $\mathrm{cm}^{-2}$ to $10.37 \mathrm{~mA} \mathrm{~cm}^{-2}$, and a reduction in FF from $68.1 \%$ to $57.5 \%$ were observed, leading to a $47 \%$ drop in PCE from $9.7 \%$ to $5 \%$. A slower degradation in the performance of the flexible OSCs with an AgNWs:AgNPs/graphene FTE, aged under the same UV exposure condition, has been observed. For example, a mild drop in $V_{o c}$ from $0.89 \mathrm{~V}$ to $0.87 \mathrm{~V}$, a smaller decrease in $J_{s c}$ from $13.4 \mathrm{~mA} \mathrm{~cm}^{-2}$ to $11.65 \mathrm{~mA} \mathrm{~cm}^{-2}$ and a lesser reduction in $\mathrm{FF}$ from $67.7 \%$ to $61.2 \%$, resulting in a relatively moderate $23 \%$ decrease in PCE from $8.15 \%$ to $6.20 \%$. It is clear that the flexible OSCs thus made have a superior UV durability as compared to that of a control OSC made with an ITO contact. The enhancement in the UV durability of the flexible OSCs is attributed to the tailored absorption of the hybrid nanostructured 
AgNWs:AgNPs/graphene FTE over the short wavelength range, as shown in Figure 3. Apart from the superior mechanical flexibility, electric conductivity and solution fabrication capability, the use of the solution-processable FTE developed in this work aids in further benefit on tailored absorption in wavelength $<380 \mathrm{~nm}$, offering an exciting option for application in low-cost large area UV durable flexible OSCs.

\section{Conclusions}

A novel solution-processable hybrid nanostructured AgNWs:AgNPs/EG FTE has been developed for application in UV durable flexible nonfullerene OSCs. The FTE possesses a high electric conductivity, an excellent mechanical flexibility and chemical stability. An average PCE of $8.15 \%$ has been realized for the flexible OSCs, made with a PBDB-T:ITIC blend layer. The flexible OSCs exhibit an excellent mechanical flexibility, retaining $>90 \%$ of the initial PCE after a test of 1000 bending cycles. Most importantly, the flexible OSCs also possess an enhanced UV durability as compared to the control OSC made with an ITO anode, taking advantage of the tailored FTE absorption in wavelength $<380 \mathrm{~nm}$. It is anticipated that FTE developed in this work offers an attractive option as an ITO alternative for application in UV durable flexible OSCs.

\section{Experimental Section}

\section{Materials}

The solution of AgNWs, with an average diameter of $80 \mathrm{~nm}$ and a length of 20-30 $\mu \mathrm{m}$, was purchased from BlueNano Company. The solution of AgNPs, with an average diameter of $60 \mathrm{~nm}$, was purchased from Sigma Aldrich. Exfoliated graphene dispersion in N-Methyl pyrrolidone solution with a concentration of $4 \mathrm{mg} \mathrm{mL}^{-1}$ was purchased from XFNANO. The lateral size of EG sheets is over the range from $0.5-3.0 \mu \mathrm{m}$, while the thickness of the graphene sheets is about 0.3-2.0 nm. PBDB-T and ITIC were purchased from Solarmer. The small molecule material Liq was obtained from e-Ray Optoelectronics Corp. PMMA, chlorobenzene and 1,8-diiodooctane were received from Sigma Aldrich. The chemical materials were used as received without further treatment and modification. 
The AgNWs:AgNPs/graphene FTEs on PET substrates were prepared by an all-solution process, the fabrication route is schematically illustrated in Figure 1(a). The PET substrates were cleaned by ultrasonication sequentially with detergent, de-ionized water, and isopropanol each for $15 \mathrm{~min}$. Then, PMMA solution (120 mg ml $\left.\mathrm{m}^{-1}\right)$ was spin-coated on PET substrates at a rotation speed of $1000 \mathrm{rpm}$ for $60 \mathrm{~s}$, followed by an annealing on a hot plate at $110{ }^{\circ} \mathrm{C}$ for $10 \mathrm{~min}$ to form a supporting layer. Subsequently, a layer of AgNPs:AgNWs was formed on the PMMA-coated PET substrates by spin coating using a mixture solution with a volume ratio of AgNPs to AgNWs of 1:8, following with an annealing at $110{ }^{\circ} \mathrm{C}$ in air for 15 min. An additional mechanical pressure was used for embedding the AgNWs in the PMMA layer. The mechanical pressure was loaded by a custom-made compressor controlled by the air pressure. The film was sandwiched between two clean glass substrates and then transferred into the compressor for embedding the AgNWs:AgNPs in the PMMA layer. Finally, EG dispersion was sprayed on the surface of the AgNPs:AgNWs layer using an airbrush system. An airbrush with a nozzle diameter of $0.3 \mathrm{~mm}$ was set in single action mode to fix a ratio of graphene to air. The inlet air pressure was set at 1.7 bar. The thickness of the EG layer was controlled by optimizing the spray parameters. The graphene dispersion was atomized into small droplets by controlling the airflow, followed by a post deposition annealing at $110{ }^{\circ} \mathrm{C}$ for $1 \mathrm{~min}$ in air to form a continuous film.

\section{Fabrication of OSCs}

PEDOT:PSS HTL was deposited on the FTE/PET substrate via a spin-coating at 3000 rpm for $60 \mathrm{~s}$, followed by an annealing at $110^{\circ} \mathrm{C}$ for $30 \mathrm{~min}$ in air. A $100 \mathrm{~nm}$ thick organic active layer was formed on the PEDOT:PSS surface by spin-coating at $1750 \mathrm{rpm}$ for $60 \mathrm{~s}$ in glove box with $\mathrm{O}_{2}$ and $\mathrm{H}_{2} \mathrm{O}$ levels $<0.1$ ppm, using a blend of PBDB-T:ITIC (1:1) in chlorobenzene and 1,8-diiodoctane (99.5:0.5 by volume) with a total concentration of $20 \mathrm{mg}$ $\mathrm{ml}^{-1}$. Finally, a $2 \times 2 \mathrm{~mm}$ sized bilayer Liq $(\sim 1.0 \mathrm{~nm}) / \mathrm{Ag}(100 \mathrm{~nm})$ cathode, defined by the shadow mask, was deposited sequentially on the active layer by thermal evaporation in a vacuum system, with a base pressure of $<10^{-6} \mathrm{~Pa}$. Liq was deposited at rate of $1.0 \AA \mathrm{s}^{-1}$, while Al was deposited at a rate of $5.0 \AA \mathrm{s}^{-1}$.

\section{Characterization}


The surface morphology of the AgNWs:AgNPs/graphene FTEs was analyzed using SEM (HITACHI S-4800) and AFM (Nanonavi SPA-400SPM) measurements. The Raman spectroscopy measurements were carried out using a Horiba LabRam spectrometer with a 473 nm laser excitation source. A 100x objective (NA 0.9) was used, having a spatial resolution < $1.0 \mu \mathrm{m}$. The optical transparency and the absorption spectra of the FTEs were measured using a UV-vis spectrophotometer (HITACHI Ue3900H). The $R_{s}$ of different FTEs was measured using a 4-point probe system (RS8, BEGA Technologies). The $J-V$ characteristics of the OSCs were measured using a calibrated AM 1.5G solar simulator (ABET Sun2000) (100 $\mathrm{mW} / \mathrm{cm}^{2}$ ). EQE spectra of the OSCs were measured using a 7-SCSpec solar cell measurement system (7-STAR Co.). The OSCs were not encapsulated, the measurements were conducted in air.

The adhesion between the graphene and the AgNWs with and without the presence of the AgNPs in the FTEs was characterized using the AFM force mapping (Nanoscope Analysis v1.80, Bruker). PFQNM-LC-A-CAL probes (Bruker) with a spring constant ranging from $0.07 \mathrm{~N} / \mathrm{m}$ to $0.1 \mathrm{~N} / \mathrm{m}$, a tip radius of curvature of $65 \mathrm{~nm}$, and a tip half-angle of $18^{\circ}$ were applied in the adhesion measurements. The adhesion measurements were conducted in vacuum to reduce the noise. Force-distance mappings were acquired using a loading force of $1.0 \mathrm{nN}$, providing an indentation range from 200 to $500 \mathrm{~nm}$. Modulus and adhesion signal channel were used to display the cell force mapping.

\section{Simulation}

Absorption spectra and distributions of the electric-field amplitude of a pure AgNW, having a diameter of $80 \mathrm{~nm}$ and a length of $3.5 \mu \mathrm{m}$, and the one grouped with ten $60 \mathrm{~nm}$-sized spherical AgNPs were calculated using Comsol Multiphysics. In the calculation, we considered incident planewave normal to the AgNW/glass substrate or AgNW:AgNPs/glass substrate, polarized perpendicular to the NW's axis. The assumption of the perfectly matched layers was used in the calculation, and symmetries were used to optimize memory and computation time. 


\section{Supporting Information}

Supporting Information is available from the Wiley Online Library or from the author.

\section{Acknowledgements}

This work was financially supported by the National Natural Science Foundation of China (61775130), Natural Science Foundation of Shanghai (19ZR1419500), Research Grants Council, University Grants Committee of Hong Kong Special Administrative Region, China, General Research Fund (GRF/12302419), Collaborative Research Fund (C5037-18GF), and Hong Kong Baptist University Inter-institutional Collaborative Research Scheme (RC-ICRS/15-16/04).

\section{Conflict of Interest}

There are no conflicts to declare.

\section{References}

(1) Dong, X. Y.; Shi, P.; Sun, L.; Li, J.; Qin, F.; Xiong, S. X.; Liu, T. F.; Jiang, X.; Zhou, Y. H. Flexible nonfullerene organic solar cells based on embedded silver nanowires with an efficiency up to $11.6 \%$. J. Mater. Chem. A. 2019, 7, 1989.

(2) Cheng, P.; Li, G.; Zhan, X.; Yang, Y. Next-generation organic photovoltaics based on non-fullerene acceptors. Nat. Photonics. 2018, 12, 131-142.

(3) Hou, J.; Inganas, O.; Friend, R. H.; Gao, F. Organic solar cells based on non-fullerene acceptors. Nat. Mater. 2018, 17, 119-128.

(4) Cheng, Y. B.; Pascoe, A.; Huang, F.; Peng, Y. Print Flexible Solar Cells. Nature. 2016, $539,488-489$.

(5) Kaltenbrunner, M.; White, M. S.; Głowacki, E. D.; Sekitani, T.; Someya, T.; Sariciftci, N. S.; Bauer, S. Ultrathin and Lightweight. Organic Solar Cells with High Flexibility. Nat. Commun. 2012, 3, 770.

(6) Li, Y.Q.; Tan, L.W.; Hao, X.T.; Ong, K.S.; Zhu, F.R.; Hung, L.S. Flexible Top-emitting Electroluminescent Devices on Polyethylene Terephthalate Substrates, Appl. Phys. Lett. 2005, $86,153508$.

(7) Wu, H.; Kong, D.; Ruan, Z.; Hsu, P. C.; Wang, S.; Yu, Z.; Carney, T. J.; Hu, L.; Fan, S.; Cui, Y. A transparent electrode based on a metal nanotrough network. Nat. Nanotechnol. 2013, $8,421$.

(8) Wang, S. L.; Zhao, Y.; Lian, H.; Peng, C.; Yang, X.; Gao, Y.; Peng, Y.; Lan, W.; Ibrahim Elmi, O.; Stiévenard, D.; Wei, B.; Zhu, F. R.; Xu, T. Towards All-solution-processed Top-illuminated Flexible Organic Solar Cells using Ultrathin Ag-modified Graphite-coated 
Polyethylene Terephthalate Substrates. Nanophotonics. 2019, 8, 297.

(9) Peng, R.; Song, W.; Yan, T. Fanady, B.; Li, Y. B.; Zhan, Q. F.; Ge, Z. y. Interface bonding engineering of a transparent conductive electrode towards highly efficient and mechanically flexible ITO-free organic solar cells. J. Mater. Chem. A. 2019, 7, 11460-11467.

(10) Kim, A.; Won, Y.; Woo, K.; Jeong, S.; Moon, J. All-Solution-Processed Indium-Free Transparent Composite Electrodes Based on Ag Nanowire and Metal Oxide for Thin-Film Solar Cells. Adv. Funct. Mater. 2014, 24, 2462-2471.

(11) Peng, C.; Chen, C.; Guo, K. P.; Tian, Z. H.; Zhu, W.; Xu, T.; Wei, B. Organic light-emitting diodes using novel embedded Al gird transparent electrodes. Physica E. 2017, $87,118$.

(12) Kim, J. Y.; Park, Y. J. Carbon nanotube/ $/ \mathrm{Co}_{3} \mathrm{O}_{4}$ nanocomposites selectively coated by polyaniline for high performance air electrodes. Scientific Reports. 2017, 7, 8610.

(13) Kim, K. S.; Zhao, Y.; Jang, H.; Lee, S. Y.; Kim, J. M.; Kim, K. S.; Ahn, J. H.; Kim, P.; Choi, J. Y.; Hong, B. H. Large-scale Pattern Growth of Graphene Films for Stretchable Transparent Electrodes. Nature. 2009, 457, 706-710.

(14) Lee, M.H. ; Chen, L.X.; Li, N.; Zhu, F.R. $\mathrm{MoO}_{3}$-induced Oxidation Doping of PEDOT:PSS for High Performing Full-solution-processed Inverted Quantum-dot Light Emitting Diodes, J. Mater. Chem. C, 2017, 5, 10555-10561.

(15) Lee, J. Y.; Connor, S. T.; Cui, Y.; Peumans, P. Solution-Processed Metal Nanowire Mesh Transparent Electrodes. Nano Lett. 2008, 8, 689-692.

(16) Yang, L.; Zhang, T.; Zhou, H.; Price, S. C.; Wiley, B. J.; You, W. Solution-Processed Flexible Polymer Solar Cells with Silver Nanowire Electrodes. ACS Appl. Mater. Interfaces. 2011, 3, 4075-4084.

(17) Zhang, Y. X.; Fang, J.; Li, W.; Shen, Y.; Chen, J. D.; Li, Y. Q.; Gu, H. W.; Pelivani, S.; Zhang, M. J.; Li, Y. F.; Tang, J. X. Synergetic Transparent Electrode Architecture for Efficient Non-Fullerene Flexible Organic Solar Cells with >12\% Efficiency. ACS Nano. 2019, 13, 4686-4694.

(18) Kim, Y.; Ryu, T. I.; Ok, K. H.; Kwak, M. G.; Park, S.; Park, N. G.; Han, C. J.; Kim, B. S.; Ko, M. J.; Son, H. J.; Kim, J. W. Inverted Layer-by-Layer Fabrication of an Ultraflexible and Transparent Ag Nanowire/Conductive Polymer Composite Electrode for Use in High-Performance Organic Solar Cells. Adv. Funct. Mater. 2015, 25, 4580-4589.

(19) Xiong, W.; Liu, H.; Chen, Y.; Zheng, M.; Zhao, Y. Kong, X.; Wang, Y.; Zhang, X.; Kong, X.; Wang, P.; Jiang, L. Highly Conductive, Air-Stable Silver Nanowire@Iongel Composite Films toward Flexible Transparent Electrodes. Adv. Mater. 2016, 28, 7167-7172.

(20) Kim, T.; Kang, S.; Heo, J.; Cho, S. ; Kim, J. W.; Choe, A.; Walker, B.; Shanker, R.; Ko, H.; Kim, J. Y. Nanoparticle-Enhanced Silver-Nanowire Plasmonic Electrodes for High-Performance Organic Optoelectronic Devices. Adv. Mater. 2018, 30, 1800659.

(21) Wei, B.; Pan, S.; Wang, T.; Tian, Z.; Chen, G.; Xu, T. Solution-processed Ag-nanowire/ZnO-nanoparticle composite transparent electrode for flexible organic solar 
cells. Nanotechnology. 2016, 27, 505208.

(22) Ye, N.; Liang, T.; Zhan, L.; Kong, Y.; Xie, S.; Ma, X.; Chen, H.; Su, H.; Xu, M. S. High-Performance Bendable Organic SolarCells With Silver Nanowire-GrapheneHybrid Electrode. IEEE J. Photovolt. 2019, 9, 214.

(23) Ricciardulli, A. G.; Yang, S.; Wetzelaer, G. A. H.; Feng, X.; Blom, P. W. M. Hybrid silver nanowire and graphene-based solution-processed transparent electrode for organic optoelectronics. Adv. Funct. Mater. 2018, 28, 1706010.

(24) Zhang, X.; Wu, J.; Liu, H.; Wang, J. ; Zhao, X. F.; Xie, Z. Y. Efficient flexible polymer solar cells based on solution-processed reduced graphene oxide-Assisted silver nanowire transparent electrode. Org. Electron. 2017, 50, 255.

(25) Liu, Z. Y.; Wu, Z. S.; Yang, S.; Dong, R. H.; Feng, X. L.; Mullen, K. Ultraflexible In-Plane Micro-Supercapacitors by Direct Printing of Solution-Processable Electrochemically Exfoliated Graphene. Adv. Mater. 2016, 28 (11), 2217-2222.

(26) Liu, H. X.; Wu, Z. H.; Hu, J. Q.; Song, Q. L.; Wu, B.; Tam H. L.; Yang, Q. Y.; Choi, W. H.; Zhu, F. R. Efficient and ultraviolet durable inverted organic solar cells based on an aluminum-doped zinc oxide transparent cathode. Appl. Phys. Lett. 2013, 103, 043309.

(27) Wang, X. Z; Zhao, C. X.; Xu, G.; Chen, Z. K.; Zhu, F. R. Degradation mechanisms in organic solar cells: Localized moisture encroachment and cathode reaction. Sol. Energy Mater. Sol. Cells. 2012, 104, 1.

(28) Vijila, C.; Ng, G. M.; Tan, M. J.; Goh, W. P.; Zhu, F. R. Imbalanced charge mobility in oxygen treated polythiophene/fullerene based bulk heterojunction solar cells. Appl. Phys. Lett. 2009, 95, 263305.

(29) Kholmanov, I. N.; Dominigues, S. H.; Chou, H. ; Wang, X. H.; Tan, C.; Kim, J. Y.; L. H. F.; Piner, R.; Zarbin, J. G. Ruoff, R. S. Reduced Graphene Oxide/Copper Nanowire Hybrid Films as High-Performance Transparent Electrodes. ACS Nano. 2013, 7, 1811-1816.

(30) Lee, M. S.; Lee, K.; Kim, S. Y.; Lee, H.; Park, J.; Choi, K. H.; Kim, H. K. Kim, D. G. Lee, D. Y.; Nam, S. W.; Park, J. U. High-Performance, Transparent, and Stretchable Electrodes Using Graphene-Metal Nanowire Hybrid Structures. Nano Lett. 2013, 13, 2814-2821.

(31) Jeong, J.; Seo, J.; Nam, S.; Han, H.; Kim, H.; Anthopoulos, T. D.; Bradley, D. D. C.; Kim, Y. Significant Stability Enhancement in High- Efficiency Polymer:Fullerene Bulk Heterojunction Solar Cells by Blocking Ultraviolet Photons from Solar Light. Adv. Sci. 2016, 3, 1500269.

(32) Kimura, H.; Fukuda, K.; Jinno, H.; Park, S.; Saito, M.; Osaka, I.; Takimiya, K.; Umezu, S.; Someya, T. High Operation Stability of Ultraflexible Organic Solar Cells with Ultraviolet-Filtering Substrate. Adv. Mater.2019, 31, 1808033.

(33) Zhang, W.D.; Lan, W.X.; Lee, M.H.; Singh, J.; Zhu, F.R. A Versatile Solution-processed $\mathrm{MoO}_{3} / \mathrm{Au}$ Nanoparticles/ $\mathrm{MoO}_{3}$ Hole Contact for High Performing PEDOT:PSS-free Organic Solar Cells, Org. Electrons, 2018, 52, 1-6. 
(34) Xu,T.; Díaz Álvarez, A.; Wei, W.; Eschimese, D.; Eliet, S.; Lancry, O.; Galopin, E.; Vaurette, F.; Berthe, M.; Wei, B.; Xu, J.; Lampin, J. F.; Pallecchi, E.; Happy, H.; Vignaud, D.; Grandidier. B. Transport mechanisms in a puckered graphene-on-lattice. Nanoscale. 2018, 10, 7519.

(35) Luo, Z. L.; Cai, Z. P.; Wang, Y. B.; Wang, Y. P,; Wang, B. B. In situ growth of silver nanowires on reduced graphene oxide sheets for transparent electrically conductive films. RSC Adv. 2016, 6, 37124.

(36) Riccardulli, A. G.; Yang, S.; Feng, X.; Blom, P. W. M. Solution-Processable High-Quality Graphene for Organic Solar Cells. ACS Appl. Mater. Interfaces. 2017, 9, 25412.

(37) Hernandez, Y.; Nicolosi, V.; Lotya, M.; Blighe, F. M.; Sun. Z.; De, S.; McGovern, I. T.; Holland, B.; Byrne, M.; Gun'Ko, Y. K.; Boland, J. J.; Niraj, P.; Duesberg, G.; Krishnamurthy, S.; Goodhue, R; Hutchison, J.; Scardaci, V.; Ferrari, A. C.; Coleman, J. N. High-yield production of graphene by liquid-phase exfoliation of graphene. Nat. Nanotechnol. 2008, 3, 563.

(38) Wang, Y. X.; Chou, S. L.; Liu, H. K.; Dou, S. X. Reduced Graphene Oxide with Superior Cycling Stability and Rate Capability for Sodium Storage. Carbon. 2013, 57, 202-208.

(39) Wang, X. J.; Zhu, C. H.; Hu, X. Y.; Xu, Q. L.; Zhao, H. P.; Meng, G. W.; Lei, Y. Highly sensitive surface-enhanced Raman scattering detection of organic pesticides based on Ag-nanoplate decorated graphene-sheets. Appl. Surf. Sci. 2019, 486, 405-410.

(40) Zhang, X.; Öberg, V. A.; Du, J.; Liu, J.; Johansson, E. M. J. Extremely Light Weight and Ultra-Flexible Infrared Light-Converting Quantum Dot Solar Cells with High Power-Per-Weight Output Using a Solution-Processed Bending Durable Silver Nanowire-Based Electrode. Energy Environ. Sci. 2018, 11, 354-364.

(41) Vosgueritchian, M.; Lipomi, D.; Bao, Z. Highly Conductive and Transparent PEDOT:PSS Films with a Fluorosurfactant for Stretchable and Flexible Transparent Electrodes. Adv. Funct. Mater. 2012, 22, 421-428.

(42) Tong, T.; Babatope, B.; Admassie, S.; Meng, J.; Akwogu, O.; Akande, W.; Soboyejo, W. O. Adhesion in organic electronic structures. J. Appl. Phys. 2009, 106, 083708.

(43) Lan, W.X.; Liu, Y.; Wu, B.; Xu, B.; Pu, H.Y.; Wei, B.; Peng, Y.; Tian, W.J.; Zhu, F.R. Effect of $\mathrm{ZnO}$ Electron Extraction Layer on Charge Recombination and Collection Properties in Organic Solar Cells, ACS Appl. Energy Mater. 2019, DOI: 10.1021-acsaem.9b01383.

(44) Wang, Y.W.; Lan, W.X.; Li, N.; Lan, Z.J.; Li, Z.; Jia, J.N.; Zhu, F.R. Stability of Nonfullerene Organic Solar Cells: From Built-in Potential and Interfacial Passivation Perspectives, Adv. Energy Mater. 2019, 9, 1900157.

(45) Wang, Y.W.; Wu, B.; Wu, Z.H.; Lan, Z.J.; Li, Y.F.; Zhang, M.J.; Zhu, F.R. Origin of Efficient Inverted Nonfullerene Organic Solar Cells: Enhancement of Charge Extraction and Suppression of Bimolecular Recombination Enabled by Augmented Internal Electric Field, $J$. Phys. Chem. Lett. 2017, 8, 5264-5271. 

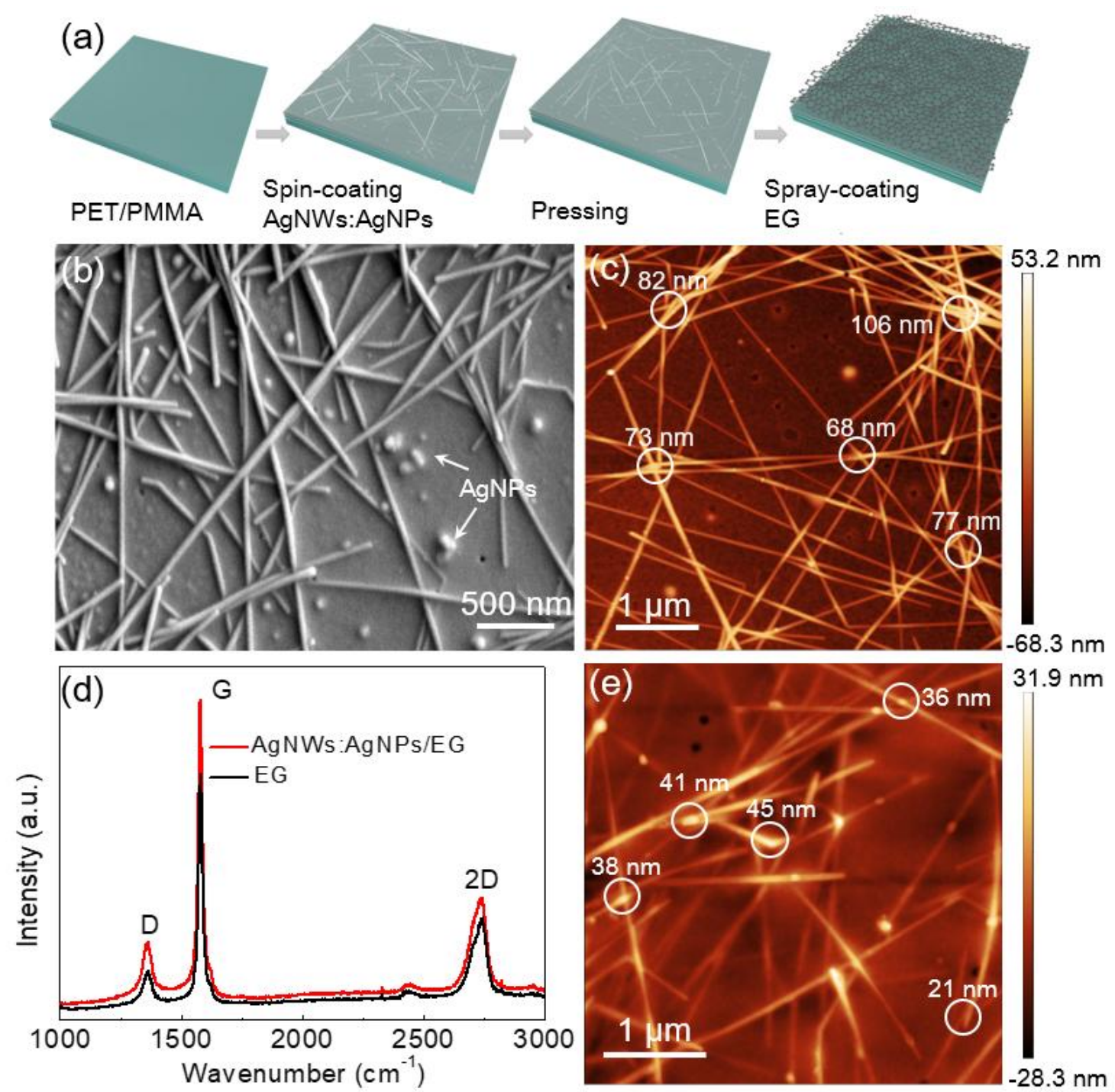

Figure 1. (a) Schematic fabrication process of AgNWs:AgNPs/EG FTE. (b) SEM and (c) AFM images measured for the surface of AgNWs:AgNPs embedded in PMMA. (d) Raman spectrum measured for the exfoliate graphene sheets and AgNWs:AgNPs/EG. (e) AFM image measured for the exfoliate graphene sheets deposited on the AgNWs:AgNPs. The white circles in the AFM images indicate the height of the overlapped AgNWs. 

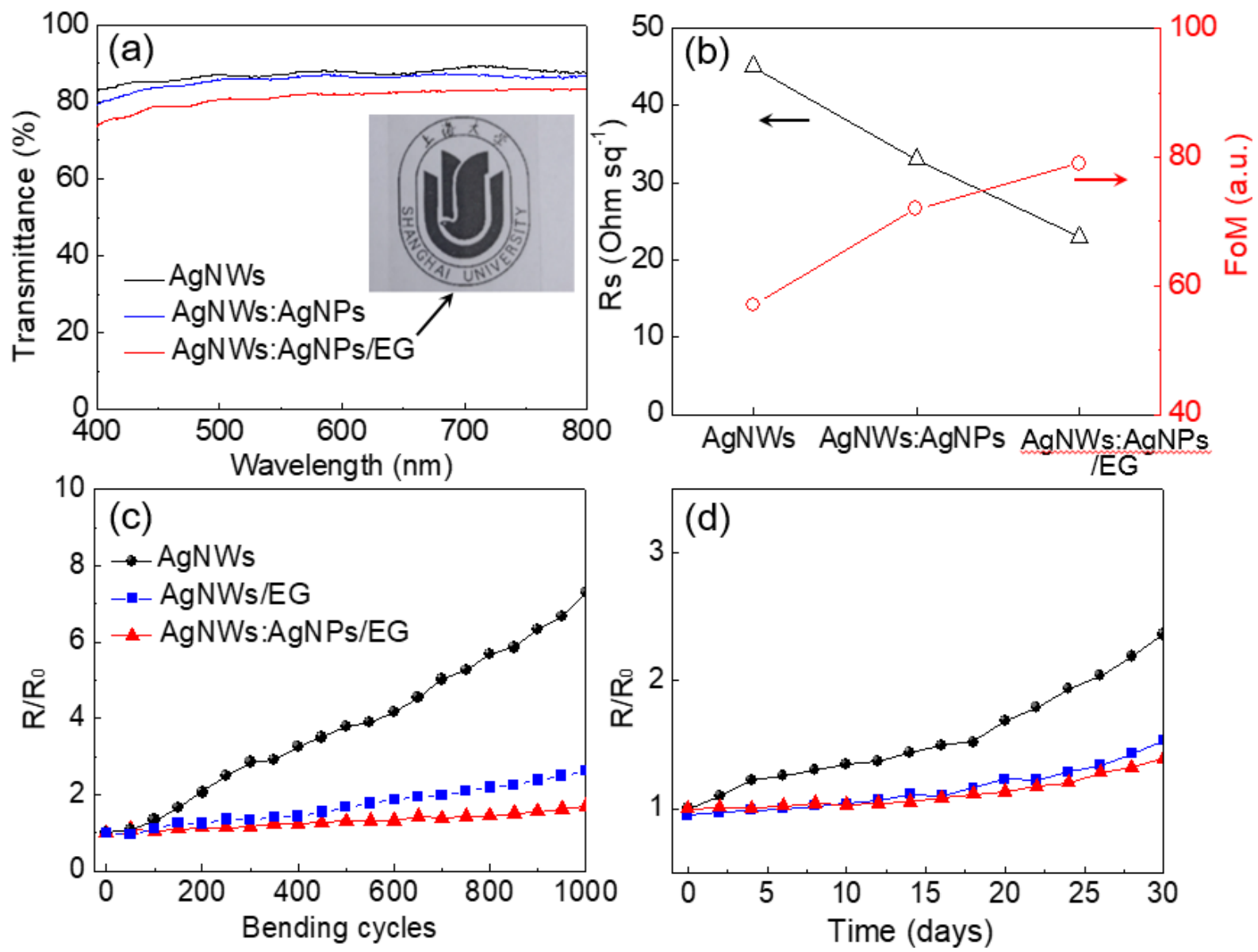

Figure 2. (a) Optical transparency spectra, and (b) sheet resistance and FoM values obtained for different FTEs of AgNWs, AgNWs:AgNPs and AgNWs:AgNPs/EG. Rs/R ratio as a function of (c) the number of bending cycles and (d) aging time for different FTEs of the bare AgNWs, AgNWs/EG and AgNWs:AgNPs/EG. 


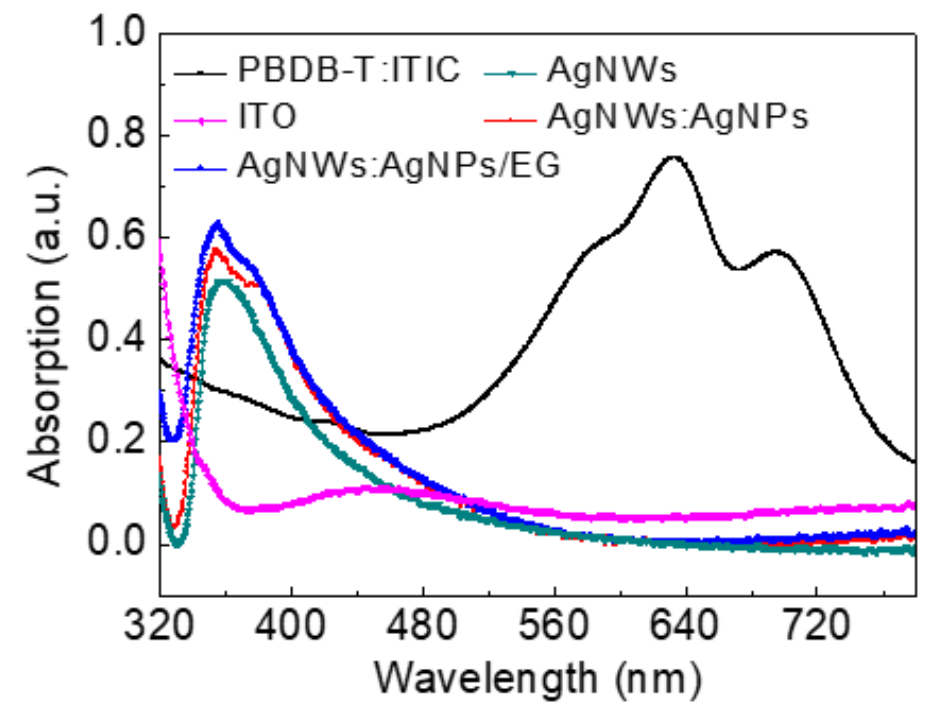

Figure 3. Absorption spectra measured for different layers of AgNWs, AgNWs:AgNPs AgNWs:AgNPs/EG, PBDB-T:ITIC and ITO used in this work. 

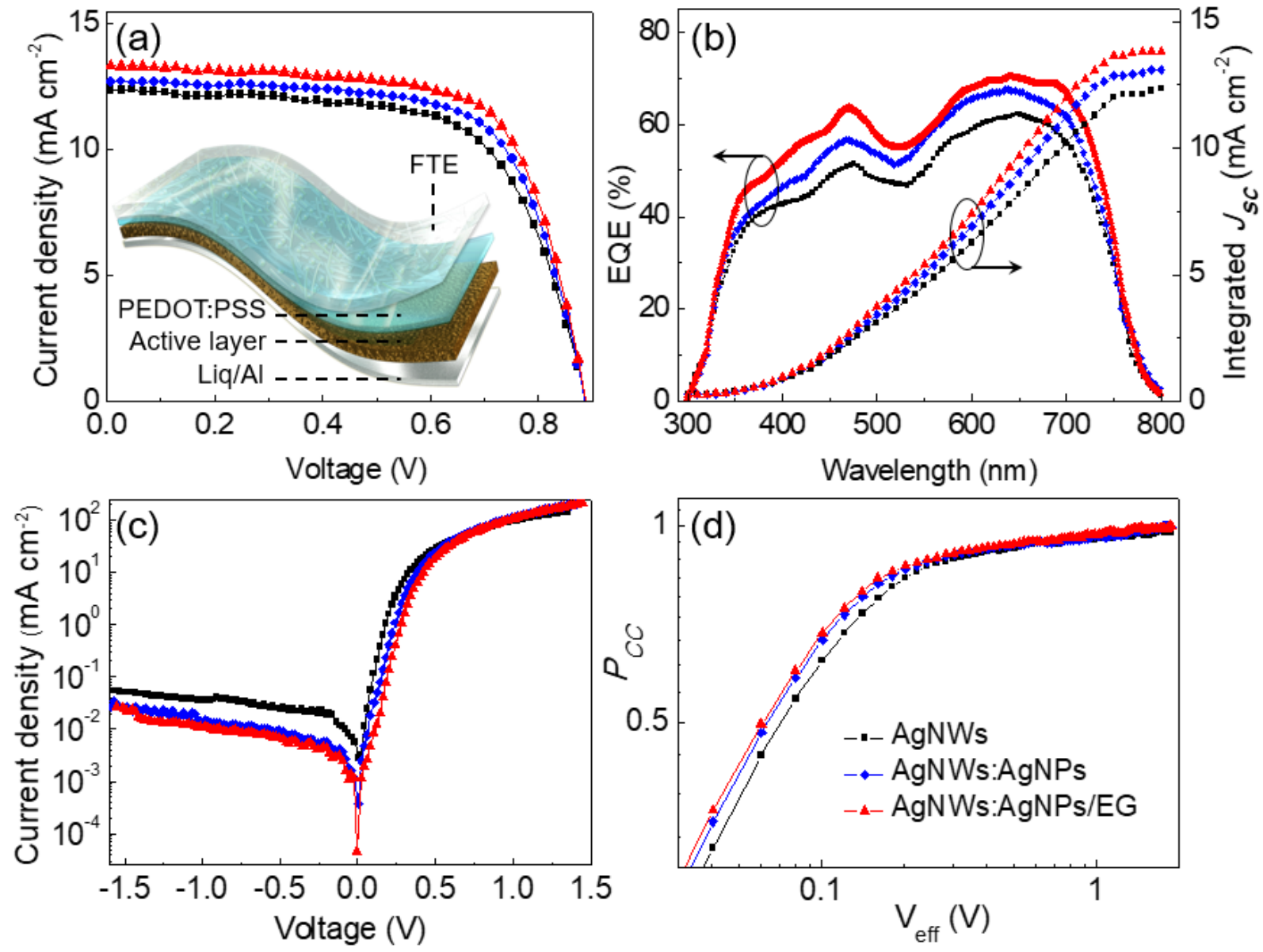

Figure 4. (a) $J-V$ characteristics, (b) EQE spectra measured, (c) dark $J-V$ characteristics and (d) charge collection efficiency $P_{\mathrm{CC}}$ characteristics for the flexible OSCs made with different hybrid nanostructured FTEs of AgNWs, AgNWs:AgNPs and AgNWs:AgNPs/EG. The integrated $J_{s c}$ calculated using EQE for the flexible OSCs with different hybrid nanostructured FTEs are also presented in (c). Inset of (a): schematic layer configuration of the flexible OSCs made with a hybrid nanostructured FTE-coated PET substrate. 

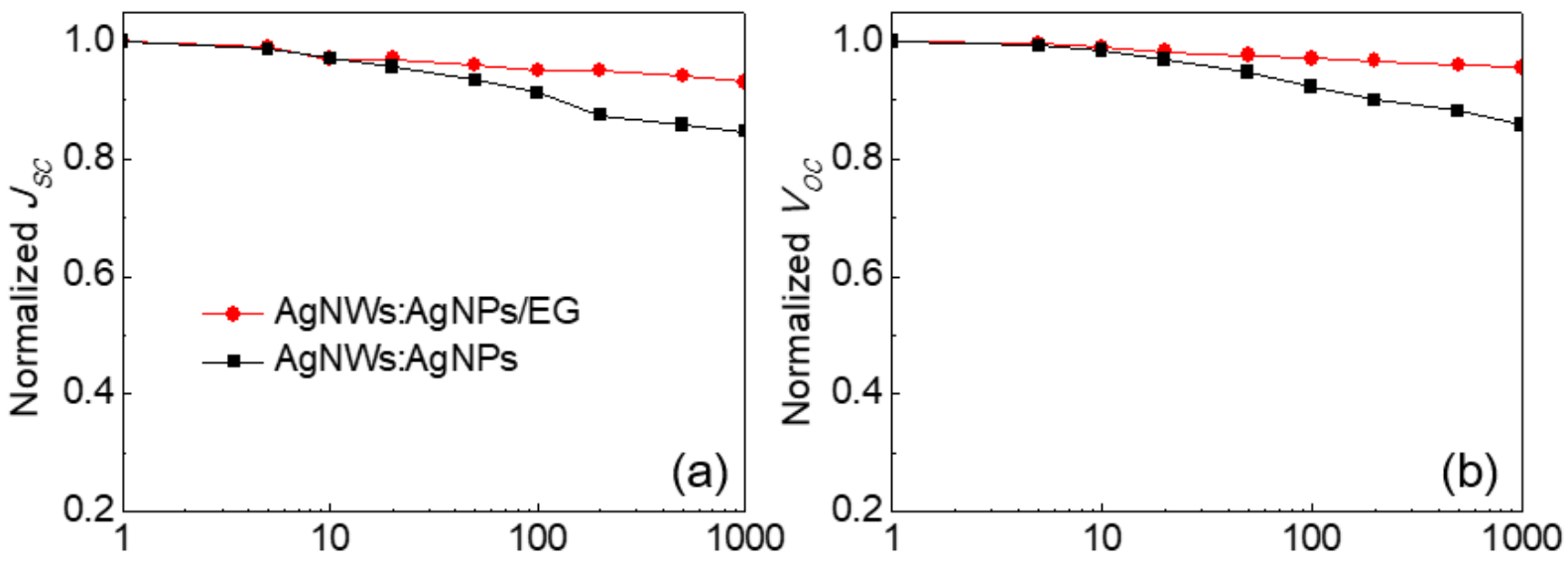

Bending cycles
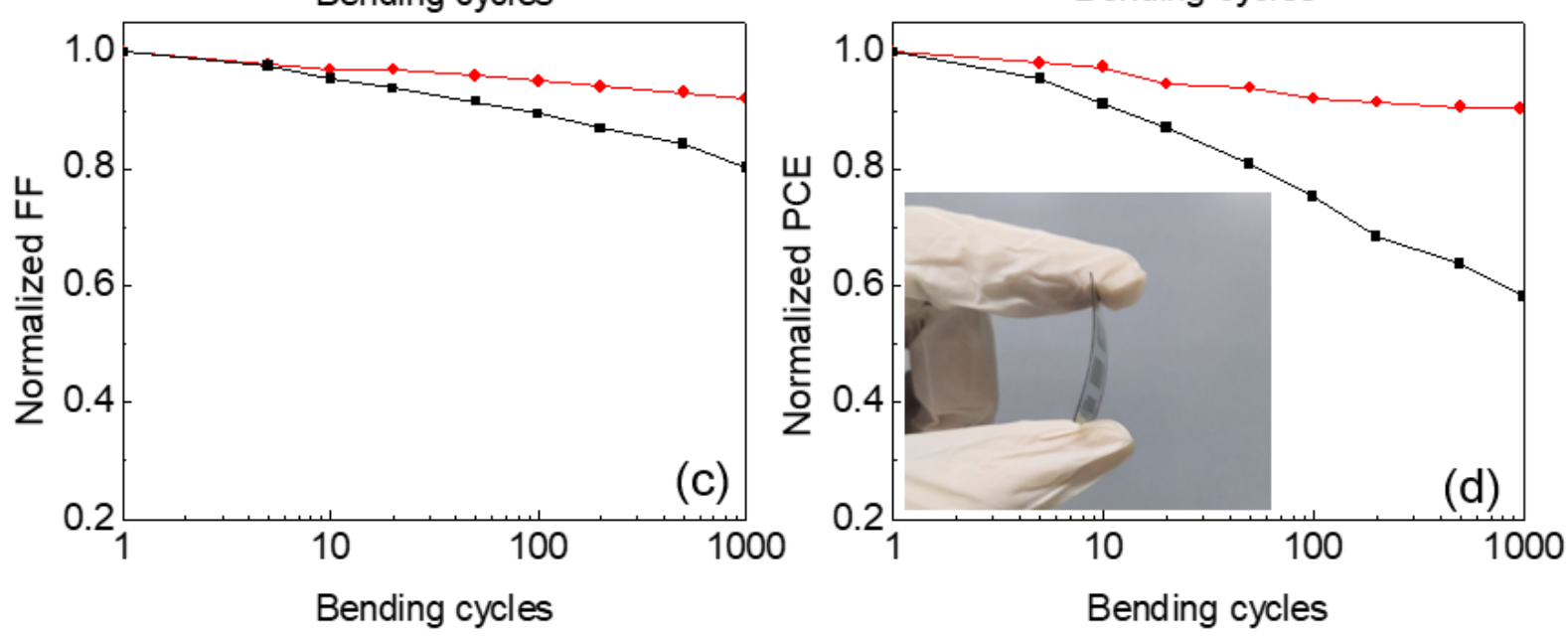

Figure 5. Normalized (a) $J_{s c}$, (b) $V_{o c}$, (c) FF and (d) PCE of the flexible OSCs with different FTEs of AgNWs:AgNPs and AgNWs:AgNPs/EG as a function of the number of the bending cycles. Inset of (a): Photograph taken for a flexible OSC. 

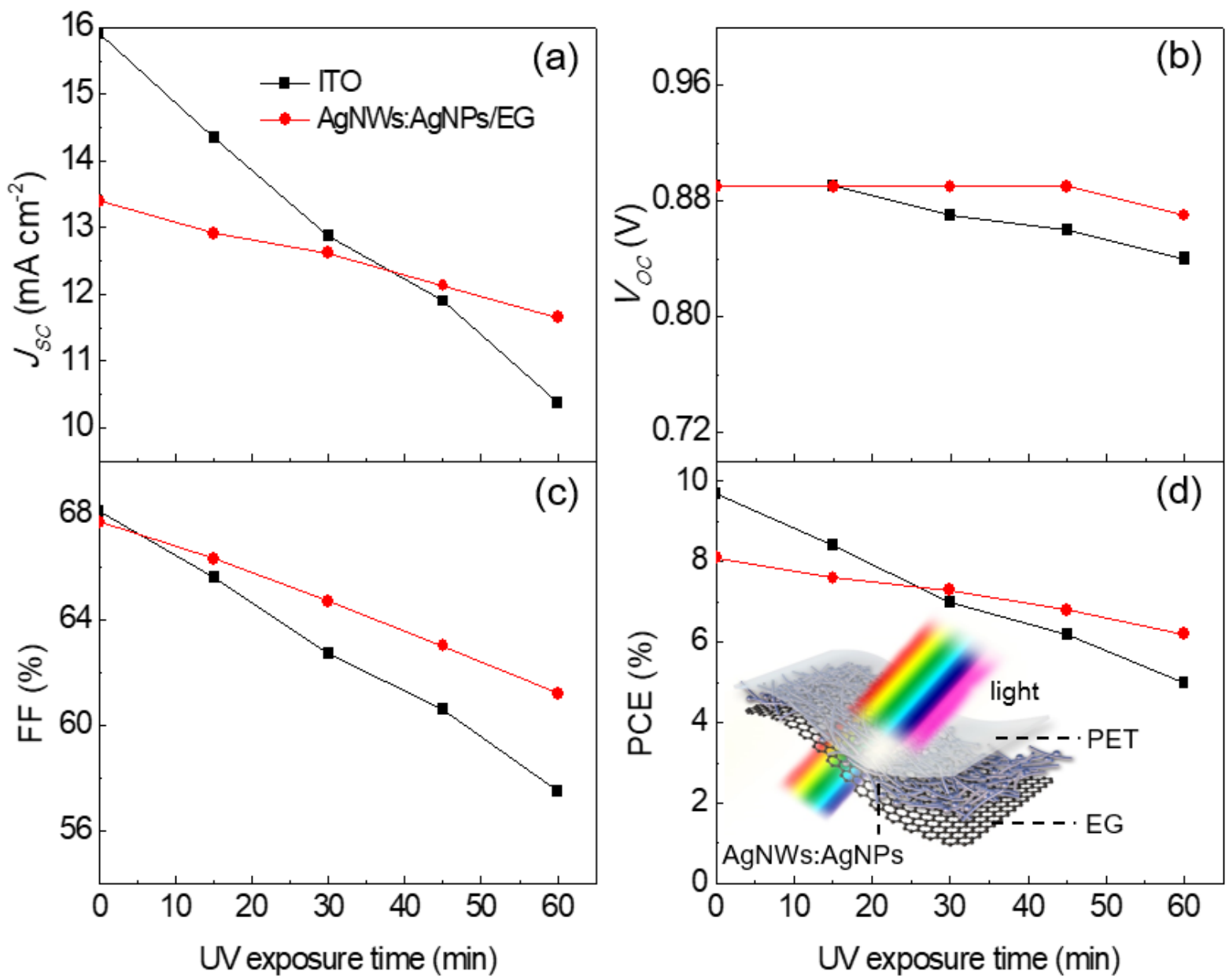

Figure 6. Evolution of (a) $J_{s c}$, (b) $V_{o c}$, (c) FF, and (d) PCE measured for a flexible OSC with an AgNWs:AgNPs/EG FTE and a control cell with an ITO anode as a function of the UV exposure time. Inset of (b): schematic of the FTE as UV filter. 
Table 1. A summary of the performance of OSCs made with different transparent electrodes, averaged from $>10$ cells.

\begin{tabular}{l|cccc}
\hline Transparent electrodes & $\begin{array}{l}\mathrm{V}_{\mathrm{oc}} \\
(\mathrm{V})\end{array}$ & $\begin{array}{c}\mathrm{J}_{\mathrm{sc}} \\
\left(\mathrm{mA} \mathrm{cm}^{-2}\right)\end{array}$ & $\begin{array}{c}\mathrm{FF} \\
(\%)\end{array}$ & $\begin{array}{c}\text { Average PCE (PCE } \\
\text { max })\end{array}$ \\
\hline AgNWs & 0.89 & 12.35 & 65.64 & $7.20(7.60)$ \\
AgNWs:AgNPs & 0.88 & 12.74 & 67.96 & $7.66(7.81)$ \\
AgNWs:AgNPs/EG & 0.89 & 13.36 & 68.55 & $8.15(8.32)$ \\
ITO & 0.89 & 15.45 & 68.89 & $9.51(9.74)$ \\
\hline
\end{tabular}




\section{Ultraviolet Durable Flexible Nonfullerene Organic Solar Cells}

Tao Xu ${ }^{1}$, Chunliu Gong ${ }^{1}$, Shuanglong Wang ${ }^{1}$, Hong Lian ${ }^{1}$, Weixia Lan ${ }^{1}$, Gaëtan Lévêque ${ }^{2}$, Bruno Grandidier ${ }^{2}$, Jerôme Plain ${ }^{3}$, Renaud Bachelot ${ }^{3}$, Bin Wei ${ }^{1 *}$, Furong Zhu ${ }^{4 *}$

${ }^{1 .}$ School of Mechatronic Engineering and Automation, Key Laboratory of Advanced Display and System Applications, Ministry of Education, Shanghai University, 200072, Shanghai, China

${ }^{2 .}$ IEMN, UMR8520, Université de Lille 1, 59652 Villeneuve d'Ascq Cédex, France

${ }^{3 .}$ Light, nanomaterials, nanotechnologies (L2n) Laboratory. Charles Delaunay Institute, CNRS. University of Technology of Troyes, 12 rue Marie Curie, F-10004 Troyes Cedex, France

${ }^{4}$ Department of Physics, Research Centre of Excellence for Organic Electronics and Institute of Advanced Materials, Hong Kong Baptist University, Kowloon Tong, Hong Kong, China

*Corresponding Authors

Email: bwei@shu.edu.cn,frzhu@hkbu.edu.hk 

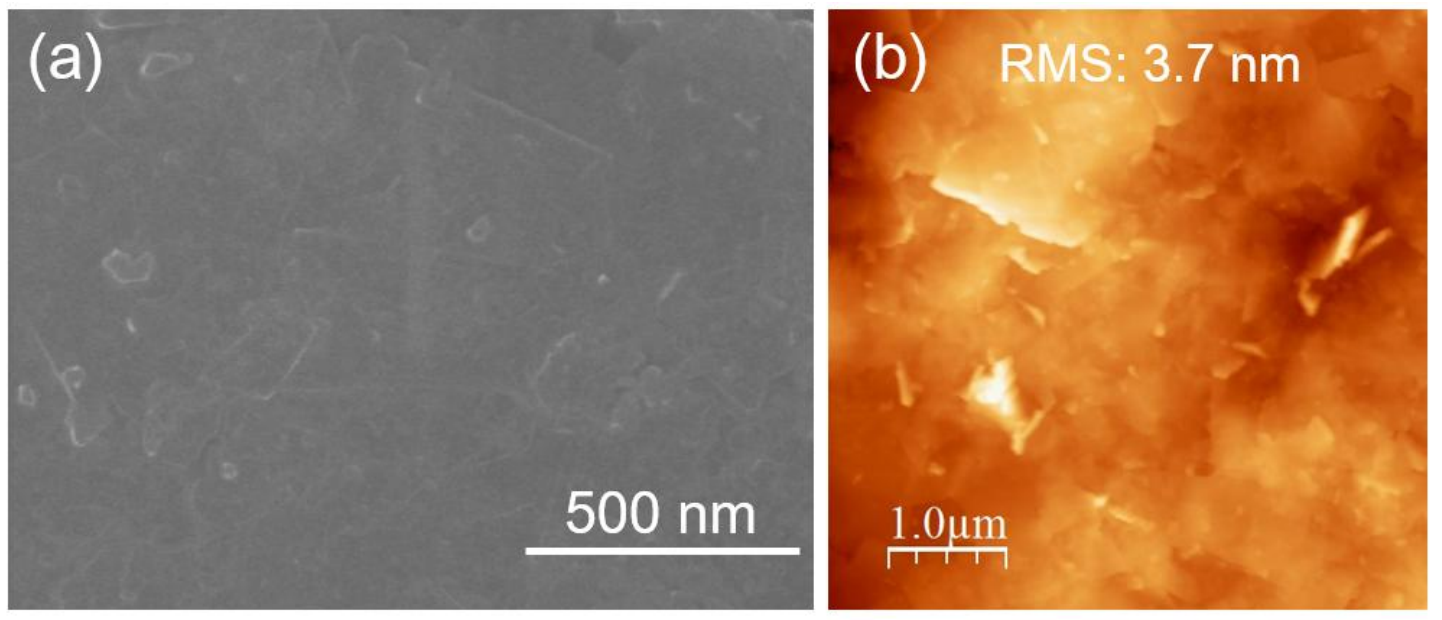

Figure S1. (a) SEM and (b) AFM images measured for the exfoliated graphene sheets formed on the ITO substrate.
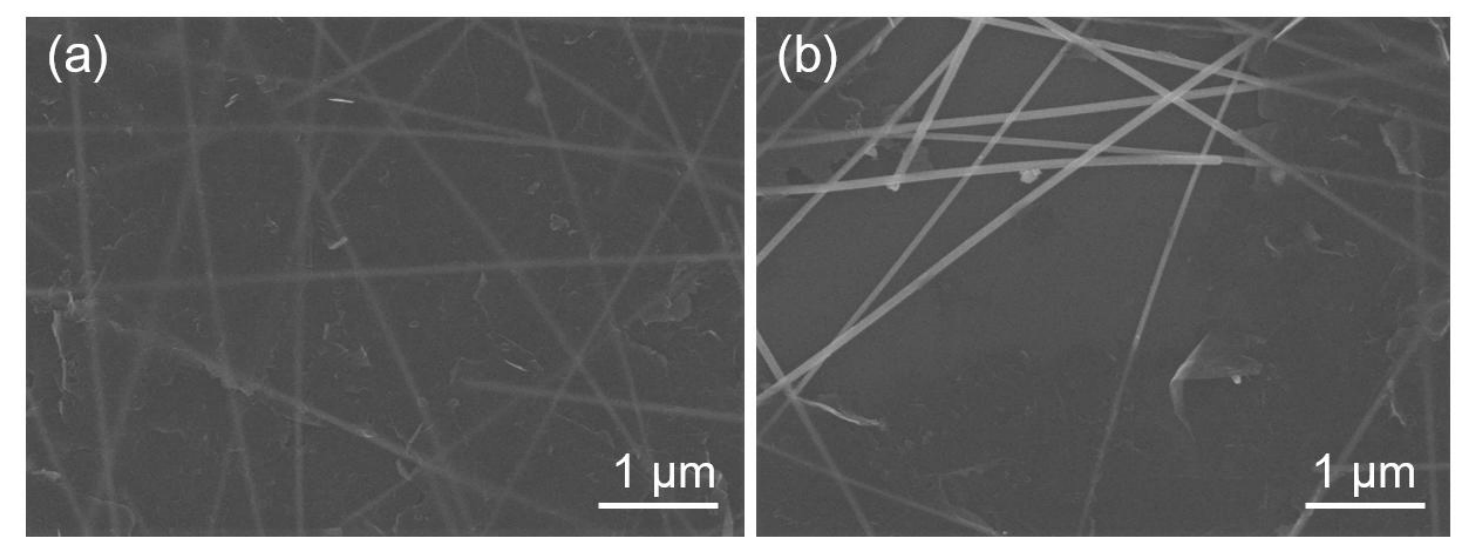

Figure S2: SEM images measured for the AgNW samples with (a) full EG coverage and (b) partial EG coverage.

(a) Contact

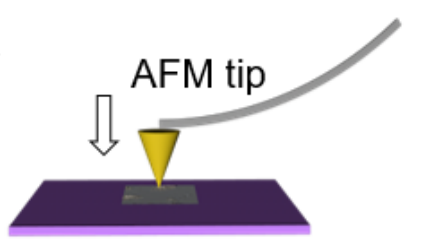

(b) Adhesion

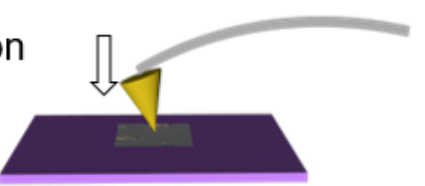

(c) Pull-off
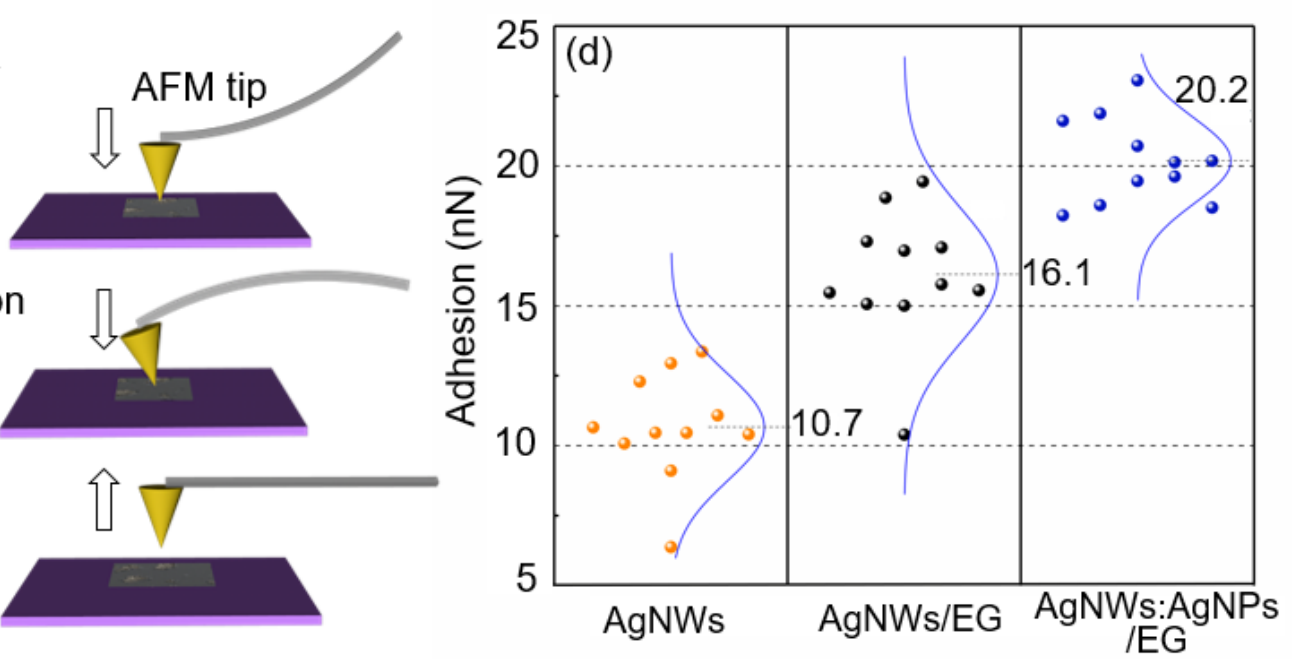

Figure S3. Adhesion measured for the AgNWs, AgNWs/EG and AgNWs:AgNPs/EG FTEs using different AFM tip-surface contact forces of (a) contact, (b) adhesion and (c) pull-off. (d) Distribution of adhesion forces used for measuring different FTEs. 

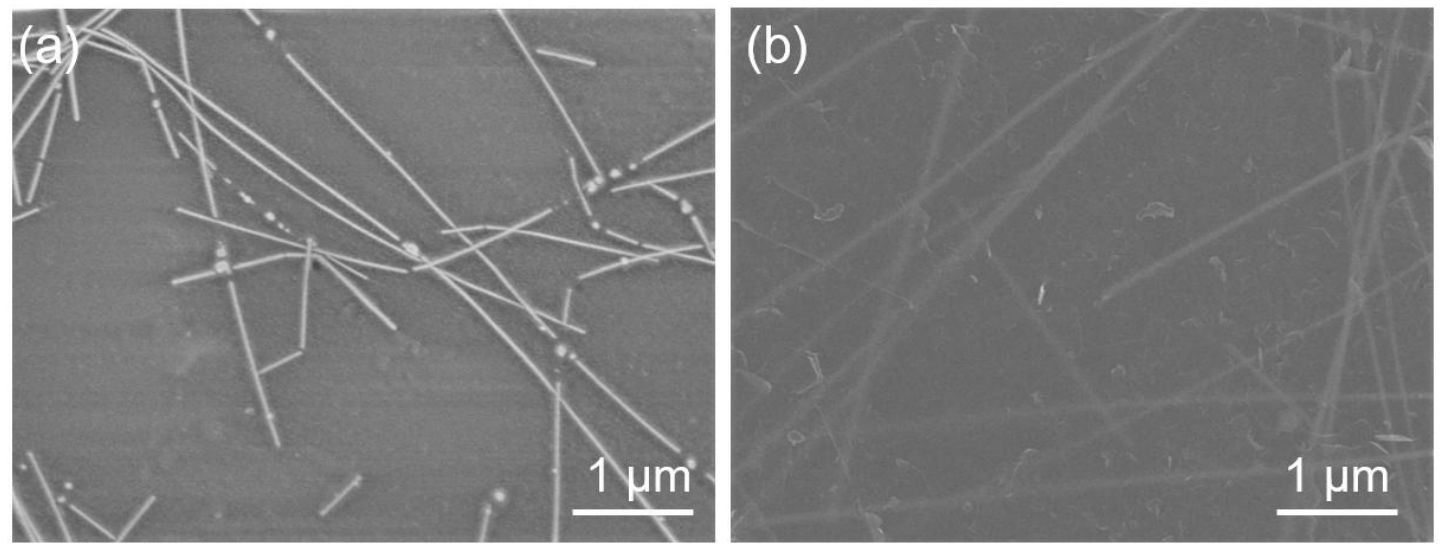

Figure S4. SEM images measured for (a) AgNWs and (b) AgNWs/EG samples exposed in air for 30 days.
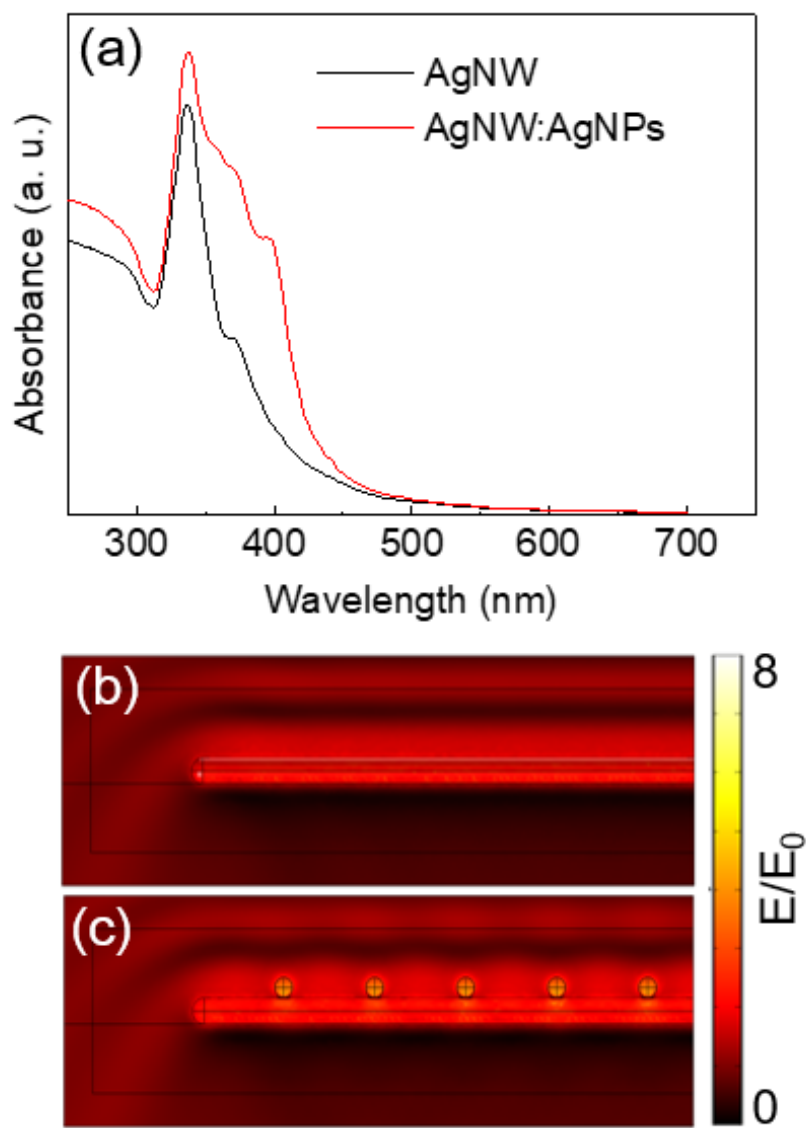

Figure S5. (a) Absorption spectra calculated for the pristine AgNW networks (black) and AgNW:AgNPs system (red). Distributions of the electric-field amplitude calculated for (b) single AgNW and (c) AgNW:AgNPs blend. 


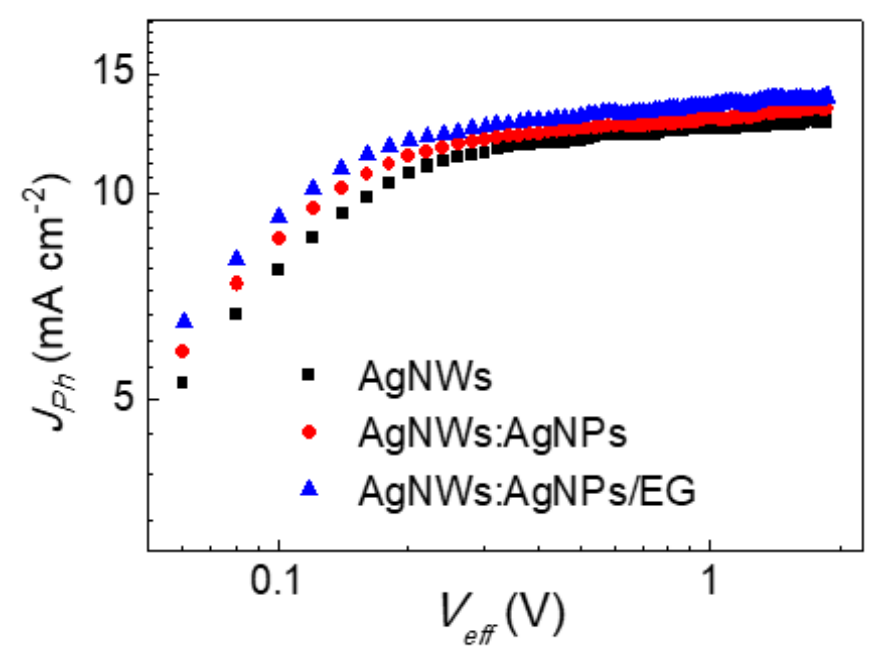

Figure S6. $J_{p h}-V_{\text {eff }}$ characteristics measured for the flexible OSCs made with different FTEs of AgNWs, AgNWs:AgNPs and AgNWs:AgNPs/EG. 